\title{
Research on Electric Field Computational Simulation of Medium Voltage Generator Insulation Structure with Air-Filled Void Defects
}

\author{
Geng $\mathrm{Li}^{1, *}$, Liang Chen ${ }^{2}$ and Benxiang $\mathrm{Wu}^{1}$ \\ ${ }^{1}$ College of Electrical Engineering, Naval University of Engineering, Wuhan 430033, China \\ ${ }^{2}$ College of Power Engineering, Naval University of Engineering, Wuhan 430033, China \\ ${ }^{*}$ Corresponding author
}

\begin{abstract}
Based on the insulation structure in stator slot of typical generators, this paper presents an analytical illustration to the fault expansion cause of turn-to-turn insulation failure through the fault example. The equivalent circuit is built up for analyzing the solid insulation layer with defects such as air bubble, and the partial discharge (PD) condition is studied according to the electric field calculation results. A 2-D finite element (FE) model of insulation structure in a $6 \mathrm{kV}$ synchronous generator stator slot is set up and solved in electrostatic field. Both the result of $\mathrm{FE}$ analysis and equivalent circuit model calculation can verify with each other, which means the electric field distribution in stator slot can be solved with accuracy in detail by using the FE method, furthermore, the conclusion can benefits the generator insulation optimizing design.
\end{abstract}

Keywords- medium voltage; synchronous generator; insulation structure; electric field calculation and simulation

\section{INTRODUCTION}

The insulation structure of power equipment needs to be well designed and tested in power industry, especially in the high voltage field. Theoretical research and engineering cases which had been put into practice shows that consideration must be given to both economical efficiency and electrical safety. And of course, safety is the number one priority. In fact, the higher voltage a power device is applied for the more cost in insulation supply, meanwhile, there needs more space for insulation structure and the volume of device would be larger. However, compared with conductive parts of power devices the material of insulation structure is usually lack of mechanical strength and heat-resistant quality, the more probability that the insulation failure occurs.

Rotating machine is widely used in power system. According to statistics, $37 \%$ of the inverter-fed machine malfunction was due to stator insulation failure [1]. Consider that generator is the source of power which is critical equipment of the system, once this insulation malfunction happens there must be overhaul downtime which may cause loss of power, and this means enormous economic loss. Researchers in relevant fields have carried out studies aiming at finding out the cause of generator stator insulation failure, and put forward targeted prevention efforts. Diako Azizi and Ahmad Gholami [2] have studied the optimizing algorithm of stator slot insulation structure designed for a high voltage (HV) generator. Their algorithm is based on the origin SNOPT package developed by Prof. Philip Gill and his colleagues. Tong Yigang [3] analyzed the PD in large generator stator bar, and he presents a qualitative explanation of insulation faults caused by PD and the corresponding repair method is proposed, which has been proved by practice. Zhang Jun and his partner evaluated the stator insulation of a $\mathrm{HV}$ synchronous generator by using FE analysis. These researches has extends the understanding of insulation malfunction cause, and provide some ideas in further study on the insulation design and protection.

As a matter of fact, there is a lot number of case study on generator insulation fault but less of them were carried out with quantitative calculation. Obviously this is far from being accurate for analysis or evaluation. In recent years, with the wide application of FE method in electromagnetic field, it can be seen that this method is an idea tool for analyzing problems coupled with complex structure. In this paper, the equivalent circuit as well as 2-D FE model is built up based on stator slot insulation structure and material properties. According to the electric field calculation results, the field distribution feature is analyzed and so as the cause of electrical insulation damage is studied.

\section{InSUlATION STRUCtURE AND MATERIAL IN STATOR SLOT}

The current-carrying conductor of generator stator is separated by the insulation structure in stator slot. The structure which consisted by several non-conductive materials is designed for achieve three main goals, they are:

- Prevent short-circuit between conductors, windings and iron core or other metal components.

- The insulation structure has a good thermal conductivity and can prevent joule heat from sharply accumulation.

- The insulation structure making the winding firmly fixed in slot and limiting the vibration or displacement, so it must be strong enough. 
In general, there are three main types of winding, randomwound windings (as shown in figure I(a)), form-wound coil windings (as shown in figure I(b)) and Roebel bar windings. Strands of Roebel bar windings transposition in axial direction which has the same cross section of form-wound coil ones. Random-wound windings are usually applied in the motor with rated voltage below $1 \mathrm{kV}$. And the other two are mainly applied in medium or large size generators. Figure I(a) and (b) shows clearly the insulation structure in slot. The main part of the insulation is called groundwall insulation which is usually made of mica, synthetic resins and glass fibers. In fact, the breakdown strength of these solid insulation materials is high up to $200 \mathrm{kV} / \mathrm{mm}$ [5], basically, there is no possibility of electrical breakdown in solid part itself. However, considering the air-filled void in the insulation structure and the relatively low breakdown strength of air, the air pocket will experience PD with high possibility. The principle would be studied in the next section.

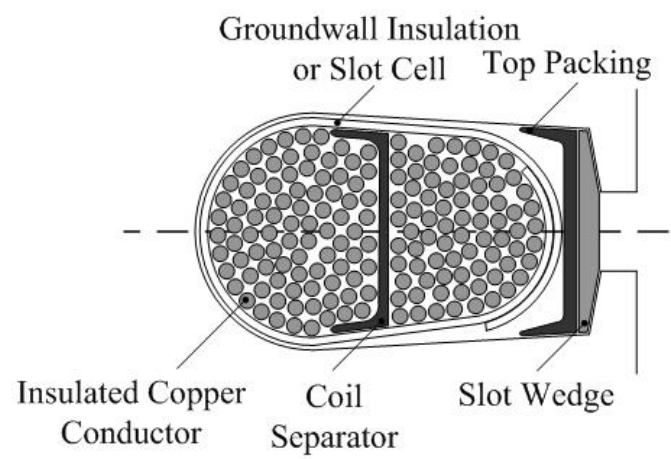

(a) Random-wound windings

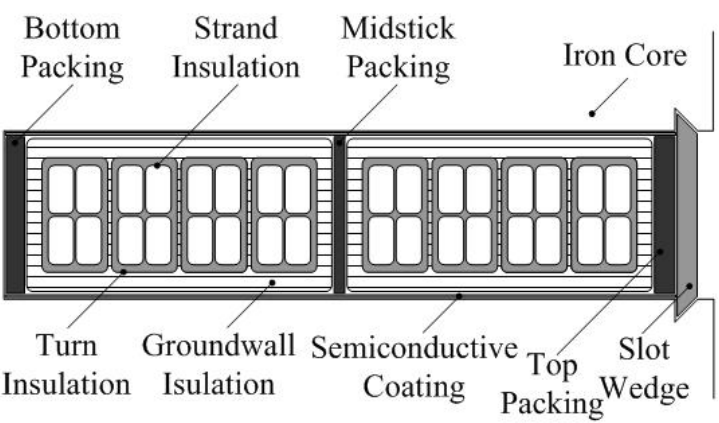

(b) Form-wound windings

FIGURE I. CROSS SECTION OF WINDINGS

\section{Equivalent Circuit of THE INSUlation StRUCtURE WITH AIR-FILLED VOID}

Figure II(a) shows the cross section of a form-wound winding in which there is a tiny air bubble mixed in the groundwall insulation. The technical term of the tiny bubble is usually known as air-filled void or air pocket. It is common for insulation aging or manufactured with low craft technique. And this phenomenon can also be treated as a sign of insulation degradation. Let $\mathrm{d}_{\mathrm{air}}$ be the width of the air pocket, and since there is an AC potential difference between winding and iron core, the air pocket can be seen as a parallel plate capacitor. The equivalent circuit of figure II(a) can be drawn as figure II(b), in which it shows a series connecting voltage divider circuit.

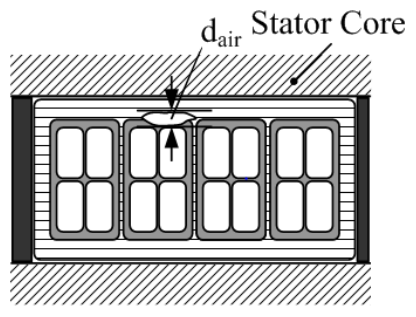

(a) Cross section of air pocket in insulation structure

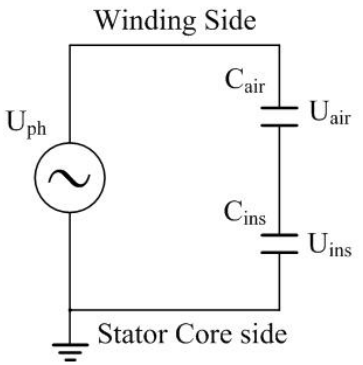

(b) Equivalent circuit

FIGURE II. EQUIVALENT MODEL OF THE INSULATION STRUCTURE WITH AIR POCKET

Suppose the voltage drop of the air pocket shows in figure II(a) in the $d_{\text {air }}$ direction is $U_{\text {air }}$, and the voltage drop of the groundwall insulation right next to it is $U_{\text {ins. }}$ They can be calculated by using equation (1) and (2) respectively,

$$
\begin{gathered}
\mathrm{U}_{\mathrm{air}}=\mathrm{C}_{\mathrm{ins}} \mathrm{U}_{\mathrm{ph}} /\left(\mathrm{C}_{\mathrm{air}}+\mathrm{C}_{\mathrm{ins}}\right) \\
\mathrm{U}_{\mathrm{ins}}=\mathrm{U}_{\mathrm{ph}}-\mathrm{U}_{\text {air }}
\end{gathered}
$$

in which $C_{a i r}$ is the capacitance of air pocket and $C_{i n s}$ is the one of groundwall insulation right next to it. Consider that the distance between winding and slot wall is $\mathrm{D}$, then the thickness of groundwall insulation containing defects is $\mathrm{D}-\mathrm{d}_{\text {air }}$. Hence $\mathrm{C}_{\text {air }}$ and $\mathrm{C}_{\text {ins }}$ can be calculated by using equation (3) and (4) respectively,

$$
\begin{gathered}
\mathrm{C}_{\text {air }}=\varepsilon_{1} \mathrm{~A} / \mathrm{d}_{\text {air }} \\
\mathrm{C}_{\text {ins }}=\varepsilon_{2} \mathrm{~A} /\left(\mathrm{D}-\mathrm{d}_{\text {air }}\right)
\end{gathered}
$$


where $\varepsilon_{1}$ and $\varepsilon_{2}$ is the permittivity of air and insulation material respectively, $\mathrm{A}$ is the equivalent area of the parallel plate capacitor which equals to the cross section area of the air void. Permittivity can be represented as,

$$
\varepsilon=\varepsilon_{\mathrm{r}} \varepsilon_{0}
$$

where $\varepsilon_{\mathrm{r}}$ is the relative dielectric constant, and the permittivity of vacuum $\varepsilon_{0}$ equals to $8.85 \times 10^{-12} \mathrm{~F} / \mathrm{m}$. The air relative dielectric constant $\varepsilon_{\mathrm{r} 1}=1$. Based on (3) to (5), equation (1) can be deduced as (6), and the electric field strength of the air pocket can be calculated as (7),

$$
\begin{gathered}
\mathrm{U}_{\mathrm{air}}=\varepsilon_{\mathrm{r} 2} \mathrm{U}_{\mathrm{ph}} \mathrm{d}_{\mathrm{air}} /\left[\mathrm{D}+\left(\varepsilon_{\mathrm{r} 2}-1\right) \mathrm{d}_{\mathrm{air}}\right] \\
\mathrm{E}_{\mathrm{air}}=\varepsilon_{\mathrm{r} 2} \mathrm{U}_{\mathrm{ph}} /\left[\mathrm{D}+\left(\varepsilon_{\mathrm{r} 2}-1\right) \mathrm{d}_{\mathrm{air}}\right] .
\end{gathered}
$$

Suppose the rated rms voltage of a generator is $6.6 \mathrm{kV}$, then the maximum phase to ground voltage is $5.38 \mathrm{kV}$. By applying a series of air width data between $0 \sim 2.59 \mathrm{~mm}$, and insulation material relative dielectric constant $\varepsilon_{\mathrm{r} 2}$ verified from 3.5 to 11 , the result of electric field strength can be plotted as the surface in figure III. As it shows, the intersection of the surface and the plane is a curve which indicates the electric field strength is $3 \mathrm{kV} / \mathrm{mm}$. When $d_{\text {air }}$ is less than $1.5 \mathrm{~mm}$, the electric field strength of the air pocket is greater than $3 \mathrm{kV} / \mathrm{mm}$. Furthermore, the greater the insulation material relative dielectric constant is, the greater electric field strength of the air pocket would be when calculated with the same $d_{\text {air }}$ data.

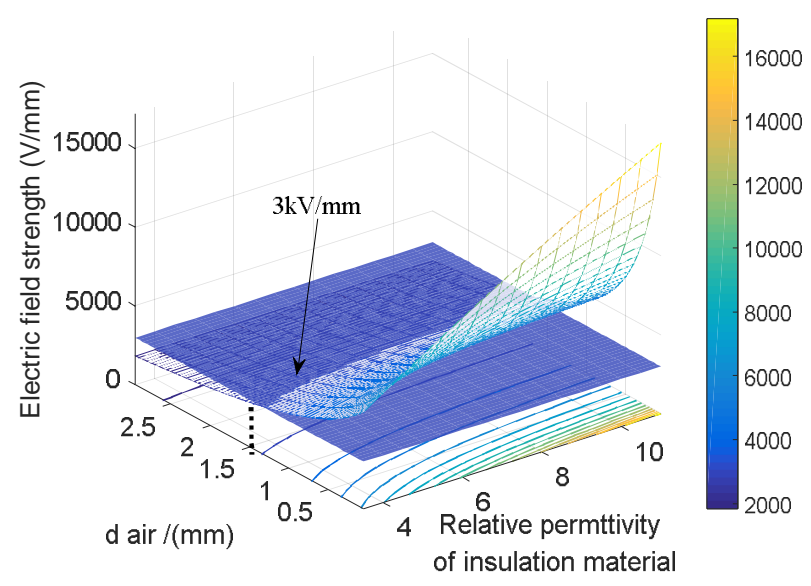

FIGURE III. ELECTRIC FIELD STRENGTH OF THE AIR POCKET

\section{FE ANALYSIS OF ELECTRICAL INSULATION STRUCTURE IN STATOR SLOT}

A 2D FE analysis model is established for electrical insulation structure in generator stator slot. As it shows in figure 4 , there is a double-layer winding which is separated by midstick packing. The rest place in the stator slot is filled with insulation structure and the detail can be referred in figure 1(b). Figure 4(b) shows the analysis model with an air pocket in insulation material which is next to the winding. The frame line marked with letter A identifies the rectangle shape air pocket.

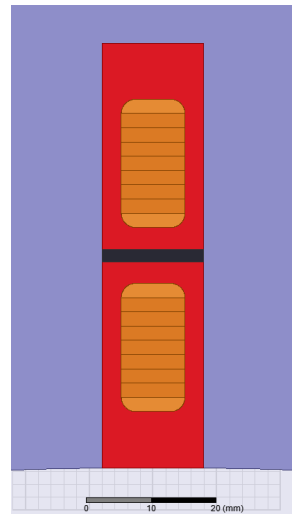

(a)Cross section of stator slot

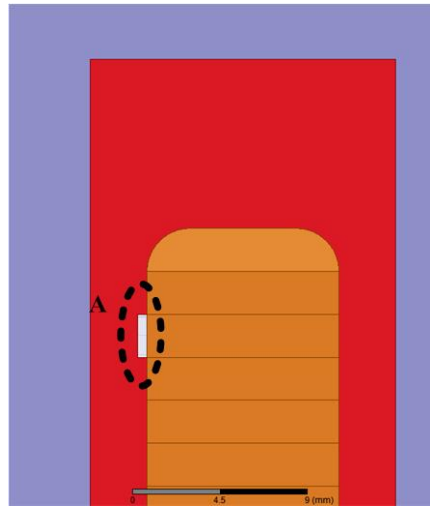

(b)Insulation structure with air pocket

\section{FIGURE IV. 2D FE ANALYSIS MODEL}

As it was mentioned above the maximum phase to ground voltage is $5.38 \mathrm{kV}$, so considering the most severe threaten of insulation structure, the voltage excitation on the winding part of the model was set to $5.38 \mathrm{kV}$. By solving Maxwell equation set using FE method, the electric field distribution can be obtained and the results were shown in figure V and VI. In figure $\mathrm{V}(\mathrm{a})$ the voltage distribution of the model is demonstrated. It is obvious that there is a dramatic decline of voltage magnitude on the air pocket. So the voltage distribution is concave in this area. The voltage drop would be larger than $1.3 \mathrm{kV}$. Meanwhile the electric field intensity has risen sharply which can be seen from figure $\mathrm{V}(\mathrm{b})$. Above all, the maximum value of electric field strength is in here.

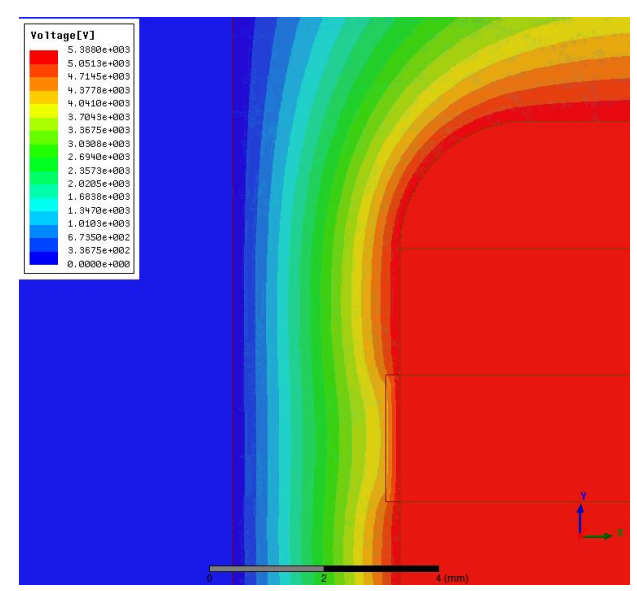

(a) voltage distribution 


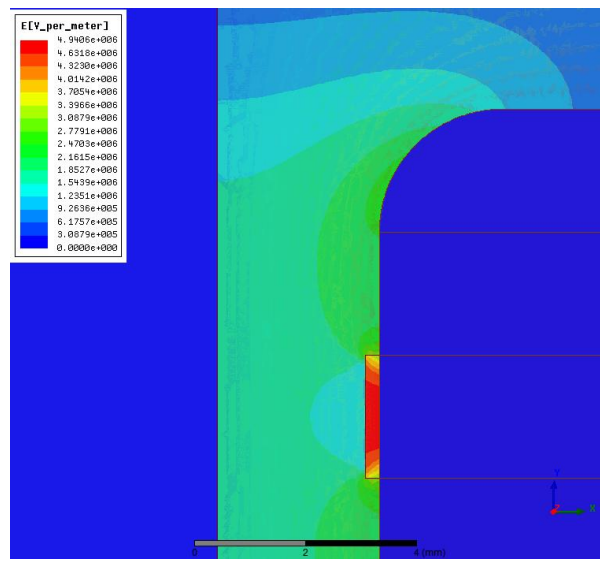

(b) Electric field distribution

\section{FIGURE V. FE METHOD CALCULATION RESULTS}

The curves in figure VI show the absolute value of electric field strength in width direction of the air pocket. It was achieved by solving a series FE model with various $d_{\text {air }}$ values. And the corresponding value to each curve is listed in table I.

TABLE I. $\quad \mathrm{d}_{\text {air }}$ VALUE CORRESPONDING TO EACH CURVE

\begin{tabular}{|c|c|c|c|c|c|c|c|c|c|c|}
\hline Curve No. & $\mathbf{1}$ & $\mathbf{2}$ & $\mathbf{3}$ & $\mathbf{4}$ & $\mathbf{5}$ & $\mathbf{6}$ & $\mathbf{7}$ & $\mathbf{8}$ & $\mathbf{9}$ & $\mathbf{1 0}$ \\
\hline dair(mm) & 0.25 & 0.5 & 0.75 & 1 & 1.25 & 1.5 & 1.75 & 2 & 2.25 & 2.5 \\
\hline
\end{tabular}

It can be seen from figure VI, the electric field strength would reach the $3 \mathrm{kV} / \mathrm{mm}$ boundary line when $d_{\text {air }}$ value is about $1.3 \sim 1.4 \mathrm{~mm}$. This result is nearly the same with which was shown in figure III.

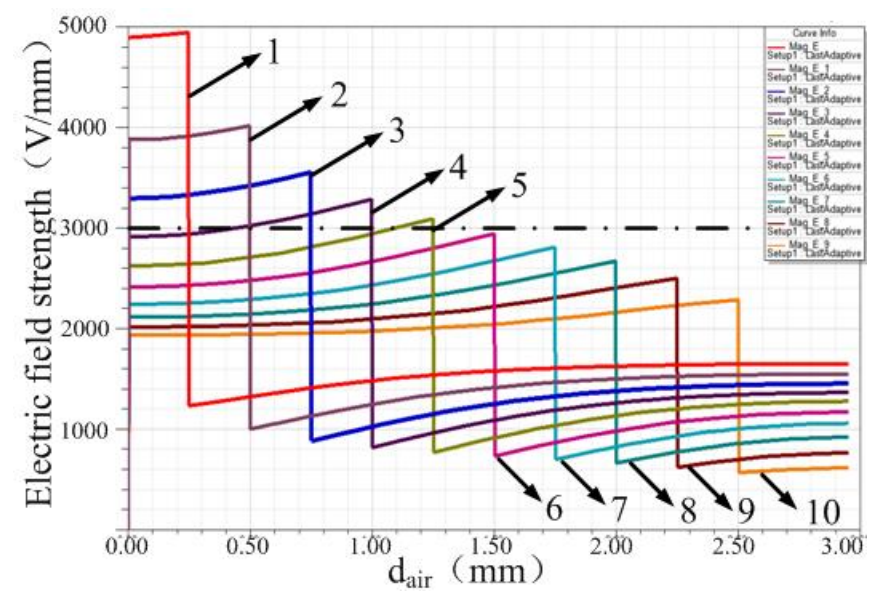

FIGURE VI. ELECTRIC FIELD STRENGTH OF WIDTH DIRECTION OF THE AIR POCKET

Specifically, the critical value of insulation structure damage is not $3 \mathrm{kV} / \mathrm{mm}$, and $3 \mathrm{kV} / \mathrm{mm}$ is far below electrical breakdown strength of most common solid insulation materials.
In fact, this is the electric breakdown strength of air in room temperature and one standard atmosphere pressure with low humidity[5]. The equivalent circuit model and FE analysis verified each other and gave proof to the conclusion that tiny air pocket in insulation structure may have reach this electrical breakdown strength. So PD would occur in this part of insulation structure which produces electric sparkle. And this may cause temperature increment in adjacent area that can accelerate the aging process of insulation material. There would be more space of cavity in insulation layer and finally leads to insulation failure. As for the case presented in this paper, the groundwall insulation failure would resulted in grounding fault of stator winding.

\section{CONCLUSION}

In this paper, the stator slot insulation structure model was established and calculated by using equivalent circuit analysis as well as FE method. A case study was given to explain the principle of insulation degradation and illustrate the process of insulation deterioration which caused by PD in air pocket.

The result of equivalent circuit analysis and FE method can verified each other, and the data proves that the equivalent circuit is accurate enough for estimate whether there would be PD in air-filled void or not. The FE model can show details of the electric field strength distribution which contains more information about the insulation structure in stator slot. The electric field strength distribution shows that there is strong electric stress in some area of the stator slot without air pocket, such as the corner of the strand in the cross-section. As for designing a better insulation structure, more studies should be carried out for optimization of the electric field distribution to make it more uniform.

\section{REFERENCES}

[1] Wu Guang ning, Zhou Kai, Gao Bo, "Mechanism and Characterization of Insulation Aging for Variable Frequency Motor", Science Press, Beijing, 2009, pp. 7-8.

[2] Diako Aziz, Ahmad Gholami, "Optimization of semiconductive coating and groundwall insulation layers in stator slot of HV generator", Electrical Power and Energy Systems. 57 (2014), pp. 384-391.

[3] Tong Yigang, "Application of partial discharge theory in analysis and treatment of burned insulation fault in generator stator", Large Electric Machine and Hydraulic Turbine. 5 (2008), pp. 19-22.

[4] Zhang Jun, Wang Shuhong, Qiu Jie, Li Haibo, et al. "Finite element analysis and evaluation of stator insulation in high voltage synchronous motor", IEEE Trans Mag 48(2) (2012), pp. 955-958.

[5] G.C. Stone, E.A. Boulter, I. Culbert, H. Dhirani, Electrical insulation for rotating machines-design, evaluation, aging, testing, and repair, 2nd ed., Wiley-IEEE Press, 2010, pp. 9-19.

[6] Application of neutral grounding in electrical utility systems-Part 1 Introduction, IEEE Std C62.92.1, 2000.

[7] Ahmad Rizal Sultan, Mohd Wazir bin Mustafa, "Ground fault protection methods of a generator stator", PRZEGLĄD ELEKTROTECHNICZNY, vol.10,pp. 225-229,2013.

[8] IEEE Guide for AC Generator Protection, IEEE Standard C37.102, 2006. 